\title{
Scalarproducts of Spinor Field Functionals and State Representations in Nonlinear Spinor Field Quantum Theory
}

\author{
H. Stumpf \\ Institut für Theoretische Physik der Universität Tübingen
}

(Z. Naturforsch. 30 a, 708-720 [1975] ; received April 5, 1975)

\begin{abstract}
In preceding papers it was shown that in nonlinear spinor theory cross-sections of elementary particle scattering processes can be calculated only if the state representations and their scalar products are explicitly known. To obtain these quantities, functional quantum theory of the nonlinear spinor field was introduced. In this paper it is demonstrated that the introduction of functional scalar products in functional quantum theory is equivalent to impose restrictions to the spinor field operator itself concerning its groundstate behaviour. Performing this, explicit state representations of spinor field states as well as corresponding scalar products can be derived, leading thus to functional quantum theories of the spinor field in dependence on the groundstate model. It follows from these considerations that a spinor field quantum theory is in principle incomplete, as long as no additional assumptions on the groundstate are made, which cannot be derived from the general dynamics of the field.
\end{abstract}

Nonlinear spinor theory treated by Heisenberg and coworkers is an approach to a unified microscopic description of matter ${ }^{1,2}$. According to Heisenberg ${ }^{3}$ for such a description it is sufficient to calculate the global observables i.e. the quantum numbers of stationary states and the S-matrix. Therefore, in nonlinear spinor theory at least these global observables have to be derived. But this task is not trivial. As the spinor field operators cannot be identified with special free matter fields of conventional quantum field theories the methods of construction of observables provided by these theories do not suffice to solve this task correctly for the nonlinear spinor field. This has been emphasized in ${ }^{4-7}$. To remove this difficulty Stumpf proposed a calculation program for the global observables ${ }^{5}$ which led to the concept of functional quantum theory ${ }^{8}$. The essential point of this approach is a map of ordinary Hilbert space of quantum field theory into a corresponding functional Hilbert space, where the observables of interest have to be calculated. In this paper we consider this map for the case of nonlinear spinor theory in more detail improving the discussion given in preceding papers ${ }^{7,9}$. It will be shown that functional quantum theory of the nonlinear spinor field is tightly connected with explicit state representations of this field and that the functional scalar products of state functionals of the spinor field depend on the special properties of the groundstate of the spinor field quantum system. By this the physical meaning of functional quantum theory becomes more transparent as well as the practical calculations can be improved. Especially it follows that the functional scalar product introduced by definition in preceding papers ${ }^{7}$ can be justified under certain well defined assumptions. Additionally the calculation technic for more general scalar products will be discussed. Summarizing the results obtained in this paper it seems that the calculational program for global observables can be performed either by postulating functional scalar products or by imposing additional conditions on the spinor field. As long as one can make no statement about the field theoretic selfconsistency of these conditions, the functional quantum theory of the nonlinear spinor field has to be considered as an appropriate mathematical framework for the global observable calculation program of this theory.

\section{Nonorthonormal Spinorial Base Sets}

In functional quantum theory of the nonlinear spinor field the physical states are described by the functionals

$$
|\mathfrak{T}(j, a)\rangle=\sum_{n=0}^{\infty} i^{n} \int \tau_{n}\left(x_{a_{1}} \ldots x_{a_{n}} \mid a\right)\left|D_{n}\left(x_{1} \ldots x_{n}\right)\right\rangle \mathrm{d}^{4} x_{1} \ldots \mathrm{d}^{4} x_{n}
$$

Reprint requests to Dr. H. Stumpf, Institut für Theoretische Physik der Universität Tübingen, D-7400 Tübingen, Auf der Morgenstelle 14. 
with

$$
\tau_{n}\left(x_{a_{1}} \ldots \underset{a_{n}}{x_{n}} \mid a\right):=\left\langle 0\left|T \Psi_{a_{1}}\left(x_{1}\right) \ldots \Psi_{a_{n}}\left(x_{n}\right)\right| a\right\rangle
$$

where $\Psi_{a}(x)$ are the Hermitean spinor field operators. The basic assumptions and definitions can be found in ${ }^{7}$. These functionals define a map from ordinary Hilbert space of the spinor field $\mathcal{H}:=\{|a\rangle\}$ into the functional Hilbert space $\mathfrak{S}:=\{|\mathfrak{I}(j, a)\rangle\}$. The expansion coefficients (1.2) of the state functional (1.1) can be considered to be projections of $|a\rangle$ on the vectors

$$
{ }_{n}\left\langle x_{1} \ldots x_{n}\right|:=\langle 0| T \Psi_{a_{1}}\left(x_{1}\right) \ldots \Psi_{\alpha_{n}}\left(x_{n}\right) .
$$

As no other vectors are known which are appropriate for the description of states of the nonlinear spinor field the set

$$
\left\{{ }_{n}\left\langle x_{1} \ldots x_{n}\right|, x_{i} \in \mathbf{M}^{4}, 1 \leqq i \leqq n, 1 \leqq n<\infty\right\}
$$

can be considered of being a natural base set of a linear metrical space in which $\mathcal{H}$ is embedded. Due to the time ordering and the physical ground state $\langle 0|$, the states (1.3) cannot expected to be orthonormal. Defining the Hermitean conjugate

one obtains therefore

$$
\left|x_{1} \ldots x_{n}\right\rangle_{n}:=\left({ }_{n}\left\langle x_{1} \ldots x_{n}\right|\right)^{+}=\bar{T} \Psi_{a_{1}}\left(x_{1}\right) \ldots \Psi_{a_{n}}\left(x_{n}\right)|0\rangle
$$

$$
{ }_{n}\left\langle x_{1} \ldots x_{n} \mid x_{1}{ }^{\prime} \ldots x_{m}{ }^{\prime}\right\rangle_{m}=g_{n m}\left(x_{1} \ldots x_{n} \mid x_{1}{ }^{\prime} \ldots x_{m}{ }^{\prime}\right)
$$

where $g_{n m}$ is unequal the unity operator, i. e. the set (1.3) is nonorthonormal. Nevertheless this set has to be used for the explicit state representation of states $|a\rangle \in \mathcal{H}$, giving

$$
|a\rangle=\sum_{n=0}^{\infty} \int \sigma_{n}\left(x_{1} \ldots x_{n} \mid a\right)\left|\underset{a_{1}}{x_{1}} \ldots x_{\alpha_{n}}\right\rangle_{n} \mathrm{~d}^{4} x_{1} \ldots \mathrm{d}^{4} x_{n} .
$$

Then the scalar product between two states $\langle b|,\langle a| \in \mathcal{H}$ can be written

with

$$
\langle b \mid a\rangle=\sum_{n=0}^{\infty} \frac{1}{(n !)^{2}} \int \tau_{n}\left(\underset{a_{1}}{\left(x_{1} \ldots x_{n}\right.} \mid b\right)^{\times} \sigma_{n}\left(x_{1} \ldots x_{n} \mid a\right) \mathrm{d}^{4} x_{1} \ldots \mathrm{d}^{4} x_{n}
$$

$$
\tau_{n}\left(x_{a_{1}} \ldots \underset{a_{n}}{x_{n}} \mid b\right)^{x}={ }_{n}\left\langle\underset{a_{1}}{x_{1} \ldots x_{n}} \mid b\right\rangle \times=\left\langle b \mid \underset{a_{1}}{x_{1}} \ldots x_{a_{n}} x_{n}\right\rangle_{n} .
$$

Usually in spaces with nonorthonormal base vectors dual base systems are introduced. For the spinor field we define them by the relations

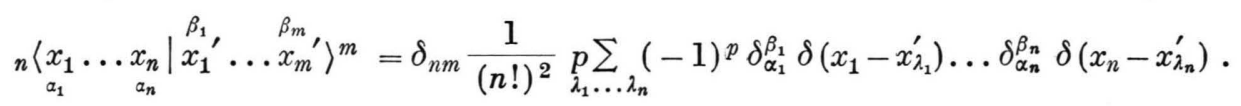

Due to the scalar product (1.7) a state $|b\rangle$ resp. $|a\rangle$ can therefore be written

$$
|b\rangle=\sum_{n=0}^{\infty} \int \tau_{n}\left(x_{a_{1}} \ldots x_{a_{n}} \mid b\right)\left|x_{1} \ldots x_{n}\right\rangle^{n} \mathrm{~d}^{4} x_{1} \ldots \mathrm{d}^{4} x_{n}
$$

and it follows from (1.9) and (1.6)

$$
\frac{1}{n !} \sigma_{n}\left(\stackrel{a_{1}}{x_{1}} \ldots{\stackrel{a_{n}}{x_{n}}}^{a} \mid a\right)={ }^{n}\left\langle\stackrel{a_{1}}{x_{1} \ldots . x_{n}^{a_{n}}} \mid a\right\rangle .
$$

Therefore, the set $\left\{\sigma_{n}(a)\right\}$ resp. $\left\{\tau_{n}(a)\right\}$ can be considered of being the co- resp. contravariant components of a state $|a\rangle$ which has been already recognized by Nishijima ${ }^{10}$ for the special case of a state expansion on a spacelike hyperplane. An analogous procedure can be performed in functional space. Defining the dual functionals

$$
|\Im(j, a)\rangle:=\sum_{n=0}^{\infty} i^{n} \int \sigma_{n}\left(\stackrel{a}{1}_{1} \ldots \stackrel{a}{n}_{n} \mid a\right)\left|D^{n}\left(\underset{a_{1}}{x_{1}} \ldots \underset{a_{n}}{x_{n}}\right)\right\rangle \mathrm{d}^{4} x_{1} \ldots \mathrm{d}^{4} x_{n}
$$


and observing the orthonormality relations ${ }^{11}$

$$
\left\langle D_{n}\left(x_{\alpha_{1}} \ldots \underset{a_{n}}{\alpha_{n}}\right) \mid D^{m}\left(x_{1}^{\beta_{1}} \ldots x_{m}^{\beta_{m}}\right)\right\rangle=\underset{\lambda_{1} \ldots \lambda_{n}}{p} \sum_{(1)^{p}}\left(-1 \delta_{\beta_{1}}^{\alpha_{1}} \delta\left(x_{1}-x_{n_{1}}^{\prime}\right) \ldots \delta_{\alpha_{n}}^{\beta_{n}} \delta\left(x_{n}-x_{\lambda_{n}}^{\prime}\right) \delta_{n m} \frac{1}{(n !)^{2}}\right.
$$

of the base functionals of $\mathfrak{S}$, one obtains immediately

$$
\langle\mathfrak{I}(j, b) \mid \subseteq(j, a)\rangle \equiv\langle b \mid a\rangle .
$$

So an isometric map of $\mathfrak{S}$ into $\mathcal{H}$ is established. But this general map is only meaningful if one is able to calculate the dual functionals (1.12). Under the very general assumptions made about the nonlinear spinor field it is not possible to derive a functional equation for the direct calculation of the dual func-

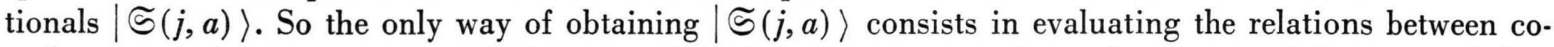
and contravariant components which are establised in linear space theory by means of the metrical fundamental tensor $g_{n m}$. Applying this to the embedding state space of $\mathcal{H}$, one obtains

$$
{ }_{n}\left\langle\underset{\alpha_{1}}{ }\left\langle x_{1} \ldots x_{\alpha_{n}} \mid a\right\rangle=\sum_{m=0}^{\infty} \int_{n m}\left(\underset{\alpha_{1}}{\left.x_{1} \ldots \underset{\alpha_{n}}{x} x_{n} \mid x_{\beta_{1}}{ }^{\prime} \ldots x_{\beta_{m}}{ }^{\prime}\right)} \sigma_{m}\left(x_{1}^{\beta_{1}} \ldots x_{m}{ }^{\beta_{m}} \mid a\right) \mathrm{d}^{4} x_{1}{ }^{\prime} \ldots \mathrm{d}^{4} x_{m}{ }^{\prime}\right.\right.
$$

a relation which has been also already derived by Nishijima ${ }^{10}$ in the special case mentioned above. Equations (1.15) can be written equivalently

$$
\begin{aligned}
\tau_{n}\left(x_{1} \ldots x_{n} \mid a\right)=\sum_{m=0}^{\infty} \int\left\langle 0\left|T \Psi_{a_{1}}\left(x_{1}\right) \ldots \Psi_{\alpha_{n}}\left(x_{n}\right) \bar{T} \Psi_{\beta_{1}}\left(x_{1}{ }^{\prime}\right) \ldots \Psi_{\beta_{m}}\left(x_{m}{ }^{\prime}\right)\right| 0\right\rangle & \\
& \times \sigma_{m}\left(x_{1}^{\beta_{1}} \ldots x_{m}{ }^{\prime} \mid a\right) \mathrm{d}^{4} x_{1}{ }^{\prime} \ldots \mathrm{d}^{4} x_{n}{ }^{\prime} \quad 1 \leqq n<\infty .
\end{aligned}
$$

Assuming the completeness relation $\sum_{b}|b\rangle\langle b|=\mathbf{1}$ to be valid for the eigenstates of the spinor field, equations (1.16) are equal to

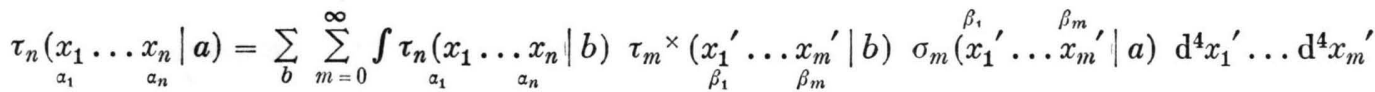

$$
\begin{aligned}
& =\sum_{b} \tau_{n}\left(\underset{a_{1}}{x_{1}} \ldots x_{\alpha_{n}} \mid b\right)\langle b \mid a\rangle=\tau_{n}\left(\underset{a_{1}}{x_{1}} \ldots x_{a_{n}} \mid a\right) .
\end{aligned}
$$

Therefore, by this procedure the system (1.16) can be reduced to an identity. But from this does not follow that the system (1.16) is meaningless at all. It is meaningless only as long as no other method for calculation of the kernel $g_{n m}$ is available than that by substitution of intermediate states. Therefore, the first task has to be, to derive an equation for $g_{n m}$. This is not possible for $g_{n m}$ itself, as this kernel is highly reducible. In the following we discuss a suitable reduction procedure. Before doing this, we observe that Eq. (1.17) can be written in functional space too. Defining the direct product of two state functionals in functional space in the usual way, one may introduce the operator

$$
G(j):=\sum_{b}|\mathfrak{T}(j, b)\rangle\langle\mathfrak{T}(j, b)| .
$$

Then Eq. (1.17) can be written

$$
|\mathfrak{I}(j, a)\rangle=G(j)|\widetilde{S}(j, a)\rangle \text {. }
$$

To remove reducibility from this equation we introduce the Normaltransform

and define

$$
|\Phi(j, a)\rangle:=e^{\frac{1}{2} j F j}|\mathfrak{I}(j, a)\rangle
$$

$$
G^{r}(j):=\sum_{b}|\Phi(j, b)\rangle\langle\Phi(j, b)|
$$

and

$$
|\Im(j, a)\rangle^{r}:=e^{-\frac{1}{2} j F j}|\Im(j, a)\rangle \text {. }
$$


Then Eq. (1.19) goes over into

$$
|\Phi(j, a)\rangle=G^{r}(j)|\Im(j, a)\rangle^{r} .
$$

Due to the definition of the Normaltransform (1.21) can be written

$G^{r}(j)=\sum_{n m} \int\left\langle 0\left|: \Psi_{a_{1}}\left(x_{1}\right) \ldots \Psi_{a_{n}}\left(x_{n}\right): \Psi_{\beta_{1}}\left(x_{1}{ }^{\prime}\right) \ldots \Psi_{\beta_{m}}\left(x_{m}{ }^{\prime}\right):\right| 0\right\rangle$

and (1.14) goes over into

$$
\times\left|D_{n}\left(x_{1}^{a_{1}} \ldots x_{n}^{a_{n}}\right)\right\rangle\left\langle D_{m}\left(x_{1}^{\beta_{1}} \ldots x_{\mathrm{m}}{ }^{\beta_{m}}\right)\right| \mathrm{d}^{4} x_{1} \ldots \mathrm{d}^{4} x_{n} \mathrm{~d}^{4} x_{1}{ }^{\prime} \ldots \mathrm{d}^{4} x_{m}{ }^{\prime}
$$

$$
\langle\Phi(j, b) \mid \Im(j, a)\rangle^{r}=\langle b \mid a\rangle .
$$

It follows from this consideration that an equivalent state representation can be given also by

with

$$
\left.|a\rangle=\sum_{n=0}^{\infty} \int \sigma_{n}^{r} \stackrel{a_{1}}{x_{1} \ldots x_{n}} \mid a\right) \underset{\alpha_{1}}{\left|x_{1} \ldots x_{n}\right\rangle_{n}^{r}} \mathrm{~d}^{4} x_{1} \ldots \mathrm{d}^{4} x_{n}
$$

and that the corresponding Normalfunctional (1.20) may be written

with

$$
\left.|\Phi(j, a)\rangle=\sum_{n=0}^{\infty} i^{n} \int \varphi_{n} \underset{\alpha_{1}}{\left(x_{1} \ldots x_{n}\right.} \mid a\right)\left|D_{n}\left(x_{1}^{a_{1}} \ldots x_{n}^{a_{n}}\right)\right\rangle \mathrm{d}^{4} x_{1} \ldots \mathrm{d}^{4} x_{n}
$$

$$
\varphi_{n}\left(x_{1} \ldots x_{a_{n}} \mid a\right):=\left\langle 0\left|: \Psi_{a_{1}}\left(x_{1}\right) \ldots \Psi_{a_{n}}\left(x_{n}\right):\right| a\right\rangle .
$$

Likewise the dual functional to (1.28) is given by

$$
\left.|\Im(j, a)\rangle^{r}:=\sum_{n=0}^{\infty} i^{n} \int \sigma_{n}^{r} \stackrel{a_{1}}{\left(x_{1} \ldots x_{n}\right.} \mid a\right)\left|D^{n}\left(\underset{a_{1}}{x_{1}} \ldots x_{a_{n}}\right)\right\rangle \mathrm{d}^{4} x_{1} \ldots \mathrm{d}^{4} x_{n} .
$$

It has to be emphasized that the normalproduct of fieldoperators introduced by (1.24) resp. (1.27) rests completely on the transformation $(1.20)$, i. e. it has so far no independent meaning. Therefore, to remove reducibility additional assumptions have to be made. This will be done in Section 3. To understand the meaning of these assumptions and to apply them for practical calculations still a further reduction step has to be performed. This will be done in Section 2 .

\section{Reduction Procedures}

The next step in the reduction procedure of Eq. (1.23) is the introduction of the socalled $\mathfrak{M}$-transform. This $\mathfrak{M}$-transform was introduced first in ${ }^{9}$ by a spectral decomposition of the $\tau_{n}$-functions. Having thus explained its physical meaning, we introduce it by a general definition which does not depend on special spectral decompositions. We introduce it by

$$
\begin{aligned}
& \left.m_{n}\left(p_{a_{1}} \ldots p_{a_{n-1}} \mid p_{a_{n}}=I_{a}\right):=\int \prod_{r=1}^{n-1} \delta\left(\sum_{\beta=1}^{r} \mathfrak{q}_{\beta}-\mathfrak{p}_{r}\right) \underset{\beta=1}{r} n q_{\beta}-n p_{r}-i \varepsilon\right)^{-1} \\
& \times \tilde{\tau}_{n}\left(\underset{a_{1}}{q_{1}} \ldots q_{a_{n}} \mid a\right) \mathrm{d}^{4} q_{1} \ldots \mathrm{d}^{4} q_{n}
\end{aligned}
$$

and by

$$
\begin{aligned}
& \tilde{\tau}_{n}\left(q_{a_{1}} \ldots q_{a_{n}} \mid a\right)=\int \delta\left(\sum_{s=1}^{n} q_{s}-p_{n}\right) \prod_{r=1}^{n-1} \delta\left(\sum_{\beta=1}^{r} q_{\lambda}-p_{r}\right)\left(\sum_{\beta=1}^{r} n q_{\beta}-n p_{r}+i \varepsilon\right)^{-1} \\
& \times(2 \pi i)^{-2 n+2} m_{n}\left(\underset{a_{1}}{p_{1}} \ldots \underset{a_{n-1}}{p_{n-1}} \mid p_{a_{n}}=I_{a}\right) \mathrm{d}^{4} p_{1} \ldots \mathrm{d}^{4} p_{n-1}
\end{aligned}
$$


where $\tilde{\tau}_{n}$ is the Fourier-transform of $\tau_{n}$ and $n$ means a timelike unitvector as is discussed in ${ }^{12}$. By mutual substitutions one proves easily the identities $\tilde{\tau}_{n} \equiv \tilde{\tau}_{n}$ and $m_{n} \equiv m_{n}$, i. e. the definitions (2.1) and (2.2) are selfconsistent. The same definitions can be applied to the contravariant representation. This gives

$$
s_{n}\left(p_{1}^{\alpha_{1}} \ldots p_{n-1}^{a_{n-1}} \mid p_{n}^{\alpha_{n}}=I_{a}\right):=\int \prod_{r=1}^{n-1} \delta\left(\sum_{\beta=1}^{r} q_{\beta}-p_{r}\right)\left(\sum_{\beta=1}^{r} n q_{\beta}-n p_{r}+i \varepsilon\right)^{-1} \tilde{\sigma}_{n}\left(q_{1}^{a_{n}} \ldots q_{n}^{a_{1}} \mid a\right) \mathrm{d}^{4} q_{1} \ldots \mathrm{d}^{4} q_{n}
$$

and

$$
\begin{aligned}
& \tilde{\sigma}_{n}\left(q_{1}^{\alpha_{1}} \ldots q_{n}^{\alpha_{n}} \mid a\right)=\int \delta\left(\sum_{s=1}^{n} q_{s}-p_{n}\right) \prod_{r=1}^{n-1} \delta\left(\sum_{\beta=1}^{r} q_{\lambda}-p_{r}\right)\left(\sum_{\beta=1}^{r} n q_{\beta}-n p_{r}-i \varepsilon\right)^{-1} \\
& \times(2 \pi i)^{-2 n+2} s_{n}\left(\left.p_{1} \ldots p_{n-1}^{\alpha_{1}}\right|_{n} ^{\alpha_{n}}=I_{a}\right) \mathrm{d}^{4} p_{1} \ldots \mathrm{d}^{4} p_{n-1} .
\end{aligned}
$$

Substitution of (2.2) into (1.1) and (2.4) into (1.12) then gives

$$
|\mathfrak{T}(j, a)\rangle=\sum_{n=0}^{\infty} i^{n} \int m_{n}\left(p_{\alpha_{1}} \ldots p_{\alpha_{n-1}}\left|\underset{\alpha_{n}}{\left.p_{n}=I_{a}\right)}\right| \mathrm{d}_{n}\left(p_{1}^{\alpha_{1}} \ldots p_{n}^{a_{n}}=I_{a}\right)\right\rangle \mathrm{d}^{4} p_{1} \ldots \mathrm{d}^{4} p_{n-1}=:|\mathfrak{M}(j, a)\rangle
$$

and

$$
|3(j, a)\rangle=\sum_{n=0}^{\infty} i^{n} \int s_{n}\left(\left.p_{1} \ldots p_{n-1}\right|^{a_{n-1}} p_{n}^{\alpha_{n}}=I_{a}\right)\left|\mathrm{d}^{n}\left(p_{\alpha_{1}} \ldots p_{a_{n}}=I_{a}\right)\right\rangle \mathrm{d}^{4} p_{1} \ldots \mathrm{d}^{4} p_{n-1}
$$

with

$$
\begin{aligned}
& \left|\mathrm{d}_{n}\left(p_{1} \ldots p_{n}\right)\right\rangle:=\int \delta\left(\sum_{s=1}^{n} q_{s}-p_{n}\right) \prod_{s=1}^{n-1} \delta\left(\sum_{\beta=1}^{r} q_{2}-p_{r}\right)\left(\sum_{\beta=1}^{r} n q_{\beta}-n p_{r}+i \varepsilon\right)^{-1} \\
& \times(n !)^{5 / 2}(2 \pi)^{-\frac{1}{2}(3 n+2)}(2 \pi i)^{\frac{1}{2}(3-3 n)} 2^{-n+1}\left|D_{n}\left(q_{1}^{\alpha_{1}} \ldots q_{n}^{a_{n}}\right)\right\rangle \mathrm{d}^{4} q_{1} \ldots \mathrm{d}^{4} q_{n}
\end{aligned}
$$

and a similar formula for the connection of $\left|d^{n}\right\rangle$ with $\left|D^{n}\right\rangle$. The scalarproduct of the $\left|d_{n}\right\rangle$ and $\mid d^{n}$ ) can be directly evaluated. One obtains by Fourier-transformation of (1.13)

$$
\begin{aligned}
& \left\langle d_{n}\left(p_{1}^{\alpha_{1}} \ldots p_{n}^{\alpha_{n}}\right) \mid d^{m}\left(p_{\beta_{1}}{ }^{\prime} \ldots p_{\beta_{m}}{ }^{\prime}\right)\right\rangle \\
& =\delta_{n}^{m} \int \delta\left(\sum_{s=1}^{n} p_{s}-p_{n}\right) \prod_{r=1}^{n-1} \delta\left(\sum_{\beta=1}^{r} \mathfrak{p}_{\lambda}-\mathfrak{q}_{r}\right)\left(\sum_{\beta=1}^{r} n q_{\beta}-n p_{r}-i \varepsilon\right)^{-1} \delta_{\beta_{1}}^{\alpha_{1}} \ldots \delta_{\beta_{n}}^{\alpha_{n}} \\
& \quad \times \frac{1}{(n !)^{2}} \sum_{\lambda_{1} \ldots \lambda_{n}}(-1)^{p} \delta\left(\sum_{s=1}^{n} \mathfrak{q}_{\lambda}-\mathfrak{p}_{r}\right) \prod_{r=1}^{n-1} \delta\left(\sum_{\beta=1}^{r} \mathfrak{q}_{\lambda_{\beta}}-\mathfrak{p}_{r}{ }^{\prime}\right)\left(\sum_{\beta=1}^{r} n q_{\lambda_{\beta}}-n p_{r}{ }^{\prime}+i \varepsilon\right)^{-1} \mathrm{~d}^{4} q_{1} \ldots \mathrm{d}^{4} q_{n} .
\end{aligned}
$$

Now it can be proven that generally only the identical permutation $\lambda_{1} \ldots \lambda_{n}=1 \ldots n$ gives a nonvanishing contribution to integrals of the kind $(2.8)^{13}$. Performing a suitable transformation of the integration variables according to ${ }^{7}$ from $(2.8)$ results

$$
\begin{aligned}
& \left\langle d_{n}\left(p_{1}^{\alpha_{1}} \ldots p_{n}\right)\right| d^{m}\left(p_{\beta_{1}}{ }^{\prime} \ldots\right. \\
& \left.\left.p_{\beta_{m}}{ }^{\prime}\right)\right\rangle=(n !)^{4}(2 \pi)^{-3 n-2} 2^{-2 n+2}(2 \pi i)^{2-2 n} \\
& \quad \times \delta_{n}^{m} \delta\left(p_{n}-p_{n}{ }^{\prime}\right) \prod_{r=1}^{n-1} \delta\left(p_{r}-p_{r}{ }^{\prime}\right)\left(n p_{r}-n p_{r}{ }^{\prime}+2 i \varepsilon\right)^{-1} \delta_{\beta_{1}}^{\alpha_{1}} \ldots \delta_{\beta_{n}}^{\alpha_{n}}
\end{aligned}
$$

and one obtains with

$$
c_{n}:=(n !)^{4}(2 \pi)^{-3 n-2} 2^{-2 n+2}(2 \pi i)^{1-n}
$$

$$
\begin{aligned}
& \langle\mathfrak{I}(j, b) \mid \mathfrak{S}(j, a)\rangle=\langle\mathfrak{M}(j, b) \mid Z(j, a)\rangle \\
& \left.\quad=\sum_{n} c_{n} \delta\left(p_{n}-p_{n}{ }^{\prime}\right) \int m_{n} \times{ }_{\alpha_{1}} p_{1} \ldots p_{\alpha_{n-1}} p_{\alpha_{n}} \mid p_{n}=I_{b}\right) s_{n}\left(p_{1} \ldots p_{n-1}^{a_{1}} \mid p_{n}^{a_{n-1}}=I_{a}\right) \mathrm{d}^{4} p_{1} \ldots \mathrm{d}^{4} p_{n-1} .
\end{aligned}
$$

Now the fourmomentum $p_{n}$ resp. $p_{n}^{\prime}$ of $m_{n}$ resp. $s_{n}$ are for any $n$ identical with the fourmomentum $J_{b}$ resp. $J_{a}$ of the states $|b\rangle$ resp. $|a\rangle$. Therefore, (2.10) can be written 


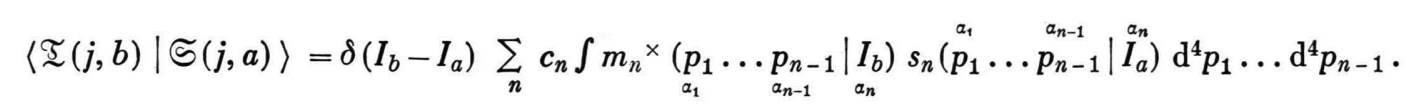

Thus having prepared the $\mathfrak{M}$-transformation we can try to reduce by this procedure Equation (1.27). Before doing this we have to investigate the connection of the general $\mathfrak{M}$-transformation (2.1) with the spectral decomposition given in ${ }^{7}$. It reads

$$
\begin{aligned}
\tilde{\tau}_{n}\left(q_{\alpha_{1}} \ldots q_{\alpha_{n}} \mid \mu_{n}\right)= & \sum_{\mu_{1} \ldots \mu_{u-1}} p \sum_{\lambda_{1} \ldots \lambda_{n}}(-1)^{p} M\left(\underset{\alpha_{\lambda_{1}}}{\mu_{1} \ldots \mu_{\alpha_{n}}}\right)\left(\frac{i}{2 \pi}\right)^{n-1}(2 \pi)^{4 n} \\
& \times \delta\left(p_{\mu_{n}}-\sum_{s=1}^{n} q_{s}\right) \prod_{s=1}^{n-1} \delta\left(\sum_{\beta=1}^{s} \mathfrak{p}_{\lambda_{\beta}}-\mathfrak{p}_{\mu_{s}}\right)\left(\sum_{\beta=1}^{s} n q_{\lambda_{\beta}}-n p_{\mu_{s}}+i \varepsilon\right)^{-1}
\end{aligned}
$$

with

$$
M\left(\mu_{a_{1}} \ldots \mu_{a_{n}}\right):=\left\langle 0\left|\Psi_{a_{1}}(0)\right| \mu_{1}\right\rangle \ldots\left\langle\mu_{n-1}\left|\Psi_{a_{n}}(0)\right| \mu_{n}\right\rangle .
$$

Substituting this decomposition into $(2.1)$ it is easily seen that for continuous mass-spectra the structure functions $M_{n}$ are identical with $m_{n}$. By this the physical interpretation of the $\mathfrak{M}$-transform is provided and can be applied immediately to the solution of Equation (1.27). For this one has to observe that in terms of the spectral decomposition (2.12) the transition from the time-ordered to Normalfunctionals is effected by substitution instead of the unreduced summation $\underset{\mu_{1} \ldots \mu_{\mu-1}}{\sum}$ by the reduced summation $\underset{\mu_{1} \ldots \mu_{\mu-1}}{\sum^{r}}$. Defining therefore the $\mathfrak{M}$-transform of $|\Phi(j, a)\rangle$ by $\mu_{1} \ldots \mu_{u-1}$

$$
|\Phi(j, a)\rangle \equiv|\mathfrak{M}(j, a)\rangle^{r}
$$

and that of $|\Im(j, a)\rangle^{r}$ by $|3(j, a)\rangle^{r}$ the scalarproduct can be written

$$
r\langle\mathfrak{M}(j, b) \mid \mathbb{Z}(j, a)\rangle^{r}=\langle b \mid a\rangle
$$

while Equation (1.27) reads

$$
|\mathfrak{M}(j, a)\rangle^{r}=G^{r}(j)|Z(j, a)\rangle^{r} .
$$

Before discussing (2.16) in full generality, we consider a special case.

\section{Generalized Free Field Bare Groundstate Model}

As has been emphasized in preceding papers functional quantum theory can be regarded in principle to be an independent selfconsistent quantum theory without connection to the corresponding field theory in the background. Accepting this point of view the metric in functional space seems to allow some flexibility which depends like in ordinary quantum theory upon the choice between possible inequivalent representations. These inequivalent representations are mainly induced by different groundstates of the theory. In this section we discuss a simple groundstate model, which of course is inspired by field theoretic models. We assume $G^{r}(j)$ to be defined by a field configuration which contains only generalized free Fermi fields and whereby the groundstate $|0\rangle$ is a bare vacuumstate, i.e. a state with no particles or antiparticles present. In this case we may write (1.24) in the form

$$
G^{r}(j)=\sum_{b}|\Phi(j, b)\rangle^{f f}\langle\Phi(j, b)|
$$

where the subscript $f$ means generalized free field states. Denoting the fourmomentum of the corresponding particles by $k$ and their spinquantum numbers by $s$ these states are given by

$$
|b\rangle^{t}:=\left|k_{1} s_{1} \ldots k_{n} s_{n}\right\rangle
$$

and the normalordered functional of (3.2) reads

$$
\left.\left.|\Phi(j, b)\rangle^{f}=\int \tilde{\varphi}_{n}\left(q_{a_{1}} \ldots q_{a_{n}} \mid k_{1} s_{1} \ldots k_{n} s_{n}\right) \mid D_{n} \stackrel{a_{1}}{\left(q_{1}\right.} \ldots \stackrel{a_{n}}{q_{n}}\right)\right\rangle \mathrm{d}^{4} q_{1} \ldots \mathrm{d}^{4} q_{n}
$$


as only $\varphi_{n}$ contributes for a $n$-particle state (3.2). For the explicit calculation of (3.3) the spectral decomposition of the spinor field occuring in (1.24) has to be fixed. We assume it to be

$$
\Psi_{a}(x):=\int \varrho\left(m^{2}\right) \Psi_{a}\left(x, m^{2}\right) \mathrm{d} m^{2} .
$$

As has been shown by Stumpf and Scheerer ${ }^{11}$ the spectral function $\varrho(m)$ can be extended to include not only real physical particles with positive masses, but also tachyons with imaginary masses. In this general case the support in momentum space covers completely the timelike as well as the spacelike regions. For brevity we do not express this distinction explicitly. Evaluating scalarproducts one has only to observe the indefiniteness of the tachyon states. By (3.4) the functionals (3.3) can be calculated. As $\tilde{\varphi}_{n} \equiv \tilde{\tau}_{n}$ for the generalized free field is valid, we obtain ${ }^{13}$ with $\varrho\left(m^{2}\right) \equiv \varrho(k)$

$$
\begin{aligned}
\tilde{\tau}_{n}{ }^{f}\left(q_{\alpha_{1}} \ldots q_{\alpha_{n}} \mid k_{1} s_{1}\right. & \left.\ldots k_{n} s_{n}\right) \\
& =\sum_{\lambda_{1} \ldots \lambda_{n}}(-1)^{p} \sum_{\lambda_{1}{ }^{\prime} \ldots \lambda_{n}{ }^{\prime}}(-1)^{p^{\prime}}(n !)^{-1 / 2} \varrho\left(k_{\lambda_{1}}\right)\left(f_{\alpha_{\lambda 1}}^{\lambda_{1}}\left(k_{\lambda_{1}} s_{\lambda_{1}}\right) \ldots \varrho\left(k_{\lambda_{n}}^{\prime}\right) f_{\alpha_{\lambda n}}^{\lambda_{n}}\left(k_{\lambda_{n}}^{\prime} s_{\lambda_{n}}^{\prime}\right)\right. \\
& \times(2 \pi)^{3 n+1} i^{n-1} \delta\left(\sum_{s=1}^{n} q_{s}-\sum_{s=1}^{n} k_{s}\right) \prod_{s=1}^{n-1} \delta\left(\sum_{\beta=1}^{s} \mathfrak{q}_{\lambda \beta}-\sum_{\beta=1}^{s} \mathfrak{f} \lambda_{\beta}^{\prime}\right)\left(\sum_{\beta=1}^{s} n q_{\lambda_{\beta}}-\sum_{\beta=1}^{s} n q_{\lambda_{\beta}}^{\prime}+i \varepsilon\right)^{-1}
\end{aligned}
$$

where $f_{\alpha}(k, s)$ are free Dirac spinors of spin $s$ and fourmomentum $k$. Substitution of (3.5) into then gives

$$
\begin{aligned}
m_{n} f\left(p_{\alpha_{1}} \ldots\right. & \left.\underset{\alpha_{n-1}}{p_{n-1}} \underset{\alpha_{n}}{\mid} p_{n}=\sum k_{i}\right)=(n !)^{-1 / 2}(2 \pi)^{3 n+1} i^{n-1}(2 \pi i)^{n-1} \\
& \quad \underset{\lambda_{1} \ldots \lambda_{n}}{\times} \sum(-1)^{p} \prod_{r=1}^{n-1} \delta\left(\sum_{\beta=1}^{r} \mathfrak{f}_{\lambda_{\beta}}-\mathfrak{p}_{r}\right)\left(\sum_{\beta=1}^{r} n k_{\lambda_{\beta}}-n p_{r}+2 i \varepsilon\right)^{-1} \varrho\left(k_{\lambda_{1}}\right) f_{\alpha_{1}}\left(k_{\lambda_{1}} s_{\lambda_{1}}\right) \ldots \varrho\left(k_{\lambda_{n}}\right) f_{\alpha_{n}}\left(k_{\lambda_{n}} s_{\lambda_{n}}\right)
\end{aligned}
$$

leading to the $\mathfrak{M}$-transform

$$
\begin{aligned}
& \left|\mathfrak{M}\left(j, k_{1} s_{1} \ldots k_{n} s_{n}\right)\right\rangle^{r} \\
& =i^{n} \int m_{n}^{\dagger}\left(p_{a_{1}} \ldots p_{\alpha_{n-1}} \mid \underset{a_{n}}{p_{n}}=\sum k_{i}\right)\left|\mathrm{d}_{n}\left(p_{1}^{a_{1}} \ldots p_{n-1}, p_{n}^{a_{n-1}}=\sum k_{i}\right)\right\rangle \mathrm{d}^{4} p_{1} \ldots \mathrm{d}^{4} p_{n-1} .
\end{aligned}
$$

Observing (3.1) Eq. (2.16) then can be written

$$
|\mathfrak{M}(j, a)\rangle^{r}=\sum_{n=0}^{\infty} \sum_{\substack{k_{1} \ldots k_{n} \\ s_{1} \ldots s_{n}}}\left|\mathfrak{M}\left(j, k_{1} s_{1} \ldots k_{n} s_{n}\right)\right\rangle^{r}{ }^{r}\left\langle\mathfrak{M}\left(j, k_{1} s_{1} \ldots k_{n} s_{n}\right) \mid \mathfrak{Z}(j, a)\right\rangle^{r}
$$

where the summations run over all possible intermediate states of the generalized free fields in consideration. The further evaluation of (3.8) depends on the properties of the spectral function $\varrho\left(m^{2}\right)$ occuring in the decomposition (3.4). We consider the case with

\section{a) Continuous Spectrum of $\varrho\left(m^{2}\right)$}

In this case the summation over intermediate states in (2.24) means an integration over the entire Minkowski space. Written in detail Eq. (3.8) then reads

$$
\begin{aligned}
& \sum_{n=0}^{\infty} i^{n} \int m_{n}^{r}\left(\underset{a_{1}}{p_{1}} \ldots \underset{a_{n-1}}{p_{n-1}}\left|\underset{\alpha_{n}}{\left.\mid p_{n}=I\right)}\right| d_{n}\left(p_{1} \ldots p_{n}=I\right)\right\rangle \mathrm{d}^{4} p_{1} \ldots \mathrm{d}^{4} p_{n-1} \\
& =\sum_{n=0}^{\infty} i^{n} \int \mathrm{d}^{4} k_{1} \ldots \mathrm{d}^{4} k_{n} \sum_{s_{1} \ldots s_{n}} m_{n}^{f} \underset{a_{1}}{f}\left(p_{1} \ldots p_{a_{n-1}} p_{n-1} \mid p_{a_{n}}=\sum k_{i}\right)\left|d_{n}\left(p_{1} \ldots p_{n}=\sum k_{i}\right)\right\rangle \mathrm{d}^{4} p_{1} \ldots \mathrm{d}^{4} p_{n-1}
\end{aligned}
$$

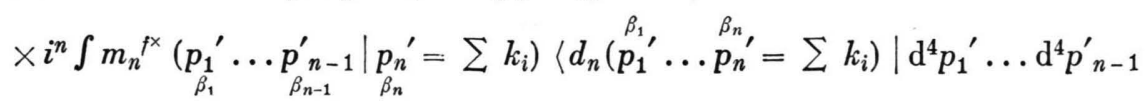

$$
\begin{aligned}
& \times \sum_{m} i^{m} \int s_{m}{ }^{r}\left(p_{\gamma_{1}}{ }^{\prime \prime} \ldots p_{\gamma_{m-1}}^{\prime \prime}{ }_{n-1} \mid p_{\gamma_{m}}{ }^{\prime \prime}=I\right)\left|\mathrm{d}^{m}\left(p_{1}^{\gamma_{1}} \ldots p_{m}{ }^{\prime \prime}=I\right)\right\rangle \mathrm{d}^{4} p_{1}{ }^{\prime \prime} \ldots \mathrm{d}^{4} p^{\prime \prime}{ }_{m-1} .
\end{aligned}
$$


By substitution of (3.6) this equation can be evaluated directly by observing that only identical permutations contribute to the integrals. Defining the spintensor

$$
\varrho_{\alpha \beta}(p):=\sum_{s} f_{\alpha}(s, p) f_{\beta}(s, p) \varrho^{2}(p)
$$

and projecting with $\left\langle d^{l}\right|$ from the left one obtains with $C_{\mathrm{n}}:=(n !)^{8}(2 \pi)^{2 n-4}(2 \pi i)^{1-n} 2^{-4 n+4}$

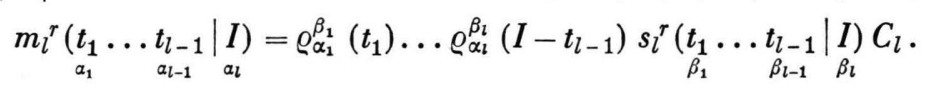

Due to the completeness relations of real particle spinors and of tachyons, the indefinite metric of a system is contained in the Dirac spinors themselves and not in the spectral function $\varrho\left(m^{2}\right)$. Therefore, without loosing the full generality of an indefinite state space we may assume $\varrho\left(m^{2}\right)$ to be positive definite about the whole range including tachyons. Then the reciprocal tensor $\varrho_{\alpha \beta}^{-1}(p)$ exists and (3.11) can be inverted to give

$$
\left.s_{l}^{r}\left(\underset{\beta_{1}}{\left(t_{1}\right.} \ldots t_{\beta_{l-1}-1}^{t_{\beta_{l}}} \mid I\right)=\varrho^{-1 \alpha_{1}}\left(t_{1}\right) \ldots \varrho^{-1 \alpha_{l}}\left(I-t_{l-1}\right) m_{l}^{r} \underset{\alpha_{1}}{\left(t_{1}\right.} \ldots \underset{a_{l-1}}{t_{l-1}} \mid I\right) C_{\alpha_{l}} C_{l}^{-1} .
$$

By this follows from (1.14) and (2.15)

$$
\begin{aligned}
& \langle\mathfrak{T}(j, b) \mid \mathfrak{S}(j, a)\rangle=\delta\left(I_{b}-I_{a}\right) \sum_{n}(n !)^{-3}(2 \pi)^{-5 n+2} 2^{2 n-2} \\
& \left.\times \int m_{n}^{r}\left(\underset{\alpha_{1}}{p_{1}} \ldots p_{\alpha_{n-1}} \mid I_{\alpha_{n}}\right) \times\left.\varrho^{-1 \alpha_{1} \beta_{1}}\left(p_{1}\right) \ldots \varrho^{-1 \alpha_{n} \beta_{n}}\left(I_{a}-p_{n-1}\right) m_{n}^{r} \underset{\alpha_{1}}{p_{1}} \ldots \underset{\alpha_{n-1}}{p_{n-1}}\right|_{\alpha_{n}} I_{a}\right) \mathrm{d}^{4} p_{1} \ldots \mathrm{d}^{4} p_{n-1}
\end{aligned}
$$

i. e. the construction of a suitable scalar product is solved in terms of the $\mathfrak{M}$-transform. We consider now the case with

\section{B) Discrete Spectrum of $\varrho\left(m^{2}\right)$}

In this case the formula (3.9) can be overtaken with the slight difference that instead of an integration over $\mathrm{d}^{4} k_{1} \ldots \mathrm{d}^{4} k_{n}$ an integration over $\mathrm{d}^{3} k_{1} \ldots \mathrm{d}^{3} k_{n}$ and a summation over the discrete masses $\left\{x_{i}\right\}$ has to be performed. This corresponds to a spectral decomposition $\Psi_{a}(x)=\sum_{i} \varrho\left(\varkappa_{i}{ }^{2}\right) \Psi_{a}\left(x, \varkappa_{i}^{2}\right)$. The evalua-
tion of formula (3.9) then leads to

$$
\begin{aligned}
& m_{l}^{r}\left(t_{\alpha_{1}} \ldots t_{\alpha_{l-1}} \mid I\right)=\sum_{\alpha_{l}} C_{\alpha_{1} \ldots x_{l}} \int \mathrm{d}^{3} z_{1} \ldots \mathrm{d}^{3} z_{l} \prod_{r=1}^{l-1} \delta\left(z_{r}-\mathrm{t}_{r}\right)\left(n z_{r}-n t_{r}-i \varepsilon\right)^{-1}(2 \pi i)^{2-2 l} \\
& \times \varrho_{\alpha_{1}}^{\beta_{1}}\left(z_{1}\right) \ldots \varrho_{\alpha_{l}}^{\beta_{l}}\left(I-z_{l-1}\right) \underset{r=1}{l-1} \delta\left(z_{r}-\mathfrak{p}_{r}^{\prime}\right)\left(n z_{r}-n p_{r}^{\prime}+i \varepsilon\right)^{-1} \delta\left(z_{l}-I\right) s_{l}^{r}\left(p_{\beta_{1}}^{\prime} \ldots p_{\beta_{l-1}}^{\prime}{ }_{l-1} \mid I\right) \mathrm{d}_{\beta_{l}}^{4} p_{1}^{\prime} \ldots \mathrm{d}^{4} p_{n-1}^{\prime}{ }_{n-1} .
\end{aligned}
$$

Changing now the discrete summations over $\varkappa_{1} \ldots \varkappa_{l}$ into integrations by introducing $\delta$-distributions of the kind $\delta\left(m^{2}-\varkappa_{i}^{2}\right)$ formula $(3.14)$ can be integrated to give

$$
\begin{aligned}
& m_{l}^{r}\left(\underset{\alpha_{1}}{\left(t_{1}\right.} \ldots t_{\alpha_{l-1}} \mid I\right) \\
& =\varrho_{\alpha_{1}}^{\beta_{1}}\left(t_{1}\right) \ldots \varrho_{\alpha_{l}}^{\beta_{l}}\left(I-t_{l-1}\right) s_{l}^{r}\left(\underset{\beta_{1}}{\left(t_{1} \ldots t_{l-1} \mid I\right)} \sum_{\beta_{l}} \sum_{x_{1} \ldots x_{l}} \delta\left(t_{1}{ }^{2}-x_{1}{ }^{2}\right) t_{1}{ }^{0} \ldots \delta\left(t_{l}{ }^{2}-x_{l}{ }^{2}\right) t_{l}{ }^{0} C_{l} 2^{l} .\right.
\end{aligned}
$$

A solution of this equation is possible only on the mass-shell by writing

$$
\begin{aligned}
& \sum_{\varkappa_{1} \ldots \varkappa_{l}} \delta\left(t_{1}^{2}-\varkappa_{1}^{2}\right) \ldots \delta\left(t_{l}^{2}-\varkappa_{l}^{2}\right) m_{l}^{r}\left(t_{a_{1}} \ldots t_{l-1} \mid I\right)
\end{aligned}
$$

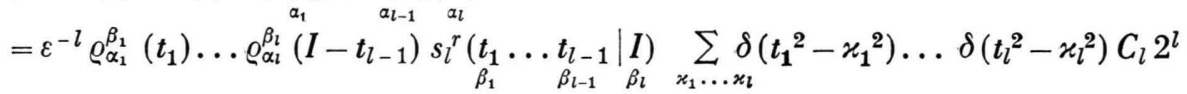

with $\varepsilon:=\delta(0)^{-1}$. Then on the mass shell

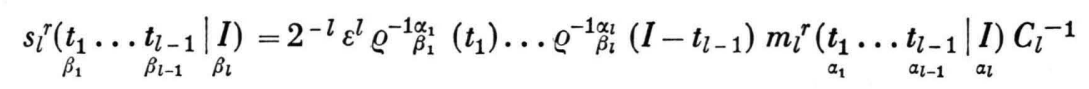

can be concluded. As in the following finally (3.17) is used only on mass shell, we may use for intermediate steps (3.17) also off mass shell without loosing generality, i. e. we define an unobservable con- 
tinuation of $s_{l}^{r}\left(t_{1} \ldots t_{l-1} \mid I\right)$ in the off mass shell region. Then formula (3.13) reads

$$
\begin{aligned}
& \langle\mathfrak{I}(j, b) \mid \mathfrak{S}(j, a)\rangle=\delta\left(I_{b}-I_{a}\right) \sum_{n} \varepsilon^{n}(n !)^{-3}(2 \pi)^{-5 n+2} 2^{n-2} \\
& \times \int m_{n}^{r}\left(\underset{a_{1}}{p_{1}} \ldots \underset{a_{n-1}}{p_{n-1}} \mid I_{a_{n}}\right) \times \varrho^{-1 a_{1} \beta_{1}}\left(p_{1}\right) \ldots \varrho^{-1 a_{n} \beta_{n}}\left(I-p_{n-1}\right) \underset{\beta_{1}}{m_{n}^{r}}\left(\underset{\beta_{1}}{p_{1}} \ldots \underset{\beta_{n-1}}{p_{n-1}} \mid \underset{\beta_{n}}{I_{a}}\right) \mathrm{d}^{4} p_{1} \ldots \mathrm{d}^{4} p_{n-1} .
\end{aligned}
$$

Expressing $m_{n}{ }^{r}$ in terms of the structure functions (2.13) we obtain for discrete spectra

$$
m_{n}^{r}\left(p_{1} \ldots p_{n-1} \mid I_{a}\right)=\sum_{x_{1} \ldots x_{m-1}}(n !) M_{n}\left(\mathfrak{p}_{1} \varkappa_{1} \ldots \mathfrak{p}_{n-1} \varkappa_{n-1} \mid a\right) \prod_{r=1}^{n-1}\left(p_{r}^{0}-\sqrt{\varkappa_{r}^{2}+\mathfrak{p}_{r}^{2}}+i \varepsilon\right)^{-1}(2 \pi)^{4 n} .
$$

Now we consider the case leading to the function scalarproduct defined in ${ }^{7}$. Identifying the masses $\varkappa_{i}$ with the masses of the intermediate states occuring in the spectral decomposition of the structure functions and assuming $\varrho\left(\varkappa_{i}{ }^{2}\right) \equiv 1,1 \leqq i<\infty$ by substitution of (3.19) into (3.18) with $\varepsilon \delta\left(I_{0}-I_{0}{ }^{\prime}\right)=\delta_{x x^{\prime}}$ and

$$
\varepsilon\left(\sqrt{\varkappa_{r}^{2}+\mathfrak{p}_{r}^{2}}-\left(\sqrt{\varkappa_{r}^{\prime 2}+\mathfrak{p}_{r}^{\prime 2}}+i \varepsilon\right)_{\mathfrak{p}_{r}=\mathfrak{p}_{r}^{\prime}}^{-1}=\delta_{\varkappa_{r} \varkappa_{r}^{\prime}}\right.
$$

we obtain finally

$$
\begin{aligned}
& \langle\mathfrak{T}(j, b) \mid \mathfrak{S}(j, a)\rangle=\langle b \mid a\rangle=\sum(2 \pi)^{3 n+2} 2^{n-2}(2 \pi i)^{n-1} \\
& \quad \times \delta\left(J_{b}-J_{a}\right) \delta_{x_{b} x_{a}}(n !)^{-1} \sum_{x_{1} \ldots x_{n-1}}^{n} \int_{n} \times\left(p_{1} \varkappa_{1} \ldots \mathfrak{p}_{n-1} \varkappa_{n-1} \mid b\right) M_{n}\left(\mathfrak{p}_{1} \varkappa_{1} \ldots \mathfrak{p}_{n-1} \varkappa_{n-1} \mid \text { a }\right) \mathrm{d}^{3} p_{1} \ldots \mathrm{d}^{3} p_{n-1} .
\end{aligned}
$$

By comparison with the physical functional scalar product defined in ${ }^{7}$ and evaluated in ${ }^{14}$ we obtain therefore the identity

$$
\langle\mathfrak{I}(j, b) \mid \mathfrak{S}(j, a)\rangle \equiv(\mathfrak{I}(j, b) \mid \mathfrak{I}(j, a)) .
$$

Thus it follows that the physical scalar product postulated in previous papers can be derived by making suitable assumptions about the groundstate behaviour of the field. From this one can conclude that postulates about the groundstate behaviour or postulates about the scalar product are equivalent and can be replaced mutually. Due to the discussions given in this paper it seems that postulates about the groundstate are more powerfull and physically transparent. Therefore, in the following we shall prefer to define the scalar product by groundstate postulates. - Finally we discuss the connection to Heisenberg's dipole ghost theory. In this theory in a first approximation the two-point vacuum function is assumed to have a discrete spectrum with an indefinite spectral function. Interpreting this function in the way performed here, the groundstate behaviour has to be described by a discrete generalized free field consisting of a free nucleon field and a dipole ghost pair. Due to the properties of the spintensor (3.10) for a proper state representation the negative norm of one of the ghostparticles results not from an indefinite spectral function, but from being a tachyon state of mass zero. So the functional scalar product postulated in preceding papers is essentially adapted to this very simple groundstate model of the Heisenberg theory.

\section{Generalized Free Field Dressed Groundstate Model}

As has been emphasized in the first section a solution of Eq. (2.16) is only possible if one is able to derive an equation for the calculation of the kernel. On the other hand the kernel of (2.16) is meaningless as long as the normal ordering procedure is defined only by the transformation (1.20). The remove this difficulty additional assumptions are required. The simplest model in this respect is the generalized free field model with bare groundstate. While the generalized free field representation (3.4) allows an independent definition of normalordering the bare groundstate leads immediately to the representation (3.6) (3.7) (3.8) which can be evaluated exactly. In this section we also use the generalized free field representation (3.4) of the spinor field operator (with respect to the vacuum-properties of the nonlinear spinor theory) but give up to the bare groundstate assumptions. Then the representation (3.6) (3.7) (3.8) of the kernel breaks down, and the kernel has to be calculated again. To do this we define

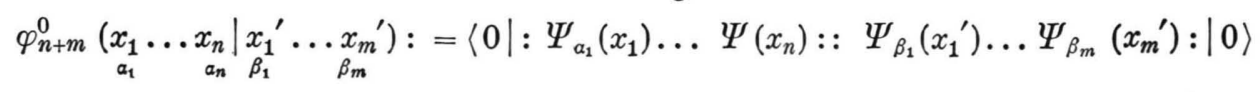


and simplify the following calculations by using the diagonal approximation of (1.24) namely

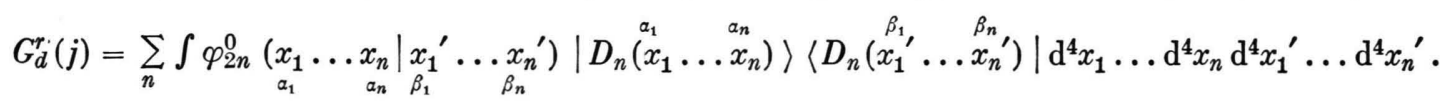

Due to (3.4) normal ordering of (4.1) is possible leading to

$$
\varphi_{2 n}^{0}\left(x_{1} \ldots x_{n} \mid x_{1}{ }^{\prime} \ldots x_{n}{ }^{\prime}\right)=\sum_{\alpha=0}^{n-1} \sum_{\substack{i_{1} \ldots i_{n} \\ j_{1} \ldots j_{n}}} F\left(x_{i_{1}} x_{j_{1}}{ }^{\prime}\right) \ldots F\left(x_{i_{\alpha}} x_{j_{\alpha}}{ }^{\prime}\right) \varphi_{2 n-2 \alpha}^{0}\left(i_{1} \ldots i_{\alpha}, j_{1} \ldots j_{\alpha}\right)
$$

where $F\left(x, x^{\prime}\right)$ is the two point function of the nonlinear spinor theory in the representation (3.4) and $\varphi_{2 n-2 \alpha}^{0}\left(i_{1} \ldots i_{\alpha}, j_{1} \ldots j_{\alpha}\right)$ means the completely normalordered expression of $(2 n-2 \alpha)$ spinorfield operators with the coordinates $\left\{x_{k}, x_{l}^{\prime}, 1 \leqq k \leqq n, 1 \leqq l \leqq n, k \neq i_{1} \ldots i_{\alpha}, l \neq j_{1} \ldots j_{\alpha}\right\}$. Considering the time ordered Schwinger functional state $|\mathfrak{I}(j, 0)\rangle$ for the vacuum by (3.4) the normalordered functional $|\Phi(j, 0)\rangle$ of the vacuum follows exactly from (1.20). Independently of any special representation of the spinor field operators for $|\mathfrak{T}(j, 0)\rangle$ a functional equation can be derived, leading by (1.20) to a functional equation for $|\Phi(j, 0)\rangle$, which can be written formally

with

$$
O(j, d)|\Phi(j, 0)\rangle=|\Phi(j, 0)\rangle
$$

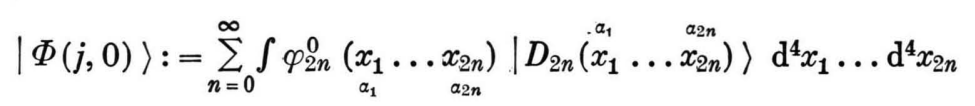

if one uses the functional integration procedure of Dammeier ${ }^{15}$. It is a question of field theoretic selfconsistency wether the functional Eq. (4.4) can be solved under the subsidiary condition (3.4) or not. We shall discuss this at the end of this section. For the following calculation we tacitly assume this selfconsistency to be satisfied. Then Eq. (4.4) can be integrated according to ${ }^{16}$. Introducing projections operators by

$$
\mathbf{P}_{2}|\Phi(j, 0)\rangle=\left|\Phi_{2}(j, 0)\right\rangle ; \Pi_{2}|\Phi(j, 0)\rangle=\left|\Phi_{r}(j, 0)\right\rangle
$$

with $|\Phi(j, 0)\rangle=\left|\Phi_{2}(j, 0)\right\rangle+\left|\Phi_{r}(j, 0)\right\rangle$, where $\left|\Phi_{2}(j, 0)\right\rangle$ represents the two point function functional, one obtains from (4.4)

and

$$
\left[\left(1-\mathbf{P}_{2} O \mathbf{P}_{2}\right)-\mathbf{P}_{2}(1-O) \Pi_{2}\left(1-\Pi_{2} O \Pi_{2}\right)^{-1} \Pi_{2}(1-O) \mathbf{P}_{2}\right]\left|\Phi_{2}(j, 0)\right\rangle=0
$$

$$
\left|\Phi_{r}(j, 0)\right\rangle=-\left(1-\Pi_{2} O \Pi_{2}\right)^{-1} \Pi_{2}(1-O) \mathbf{P}_{2}\left|\Phi_{2}(j, 0)\right\rangle \text {. }
$$

Representing the two point functional by

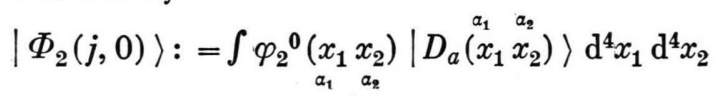

we assume (4.6) to be solved and identify $F\left(x, x^{\prime}\right) \equiv \varphi_{2}\left(x, x^{\prime}\right)$. Then we have to consider only relation (4.7). Performing a Neumann expansion of the kernel of (4.7) we obtain for the normalordered functions of (4.5)

$$
\varphi_{2 n}^{0}\left(x_{1} \ldots x_{2 n}\right)=\sum_{l=0}^{\infty}(-1)^{l+1}\left\langle D_{2 n}\left(x_{1} \ldots x_{2 n}\right)\left|\left(\Pi_{2} O \Pi_{2}\right)^{l}(1-O) \mathbf{P}_{2}\right| \Phi_{2}\right\rangle .
$$

Defining the kernel

$$
\begin{aligned}
K_{2 n}\left(x_{1} \ldots x_{n} \mid x_{1}{ }^{\prime} \ldots x_{n}{ }^{\prime}\right): & \\
& =\sum_{l=0}^{\infty}(-1)^{l+1} \sum_{\alpha=0}^{n-2} \sum_{\substack{i_{1} \ldots i_{\alpha} \\
j_{1} \ldots j_{\alpha}}} F\left(x_{i_{1}} x_{j_{1}}{ }^{\prime}\right) \ldots F\left(x_{i_{\alpha}} x_{j_{\alpha}}{ }^{\prime}\right)\left\langle D_{2 n-2 \alpha}\left(i_{1} \ldots i_{\alpha} j_{1} \ldots j_{\alpha}\right)\left|\left(\Pi_{2} O \Pi_{2}\right)^{l}(1-O) \mathbf{P}_{2}\right| \Phi_{2}\right\rangle
\end{aligned}
$$

by substitution of (4.9) into (4.3) the relation

$$
\varphi_{2 n}^{0}\left(x_{1} \ldots x_{n} \mid x_{1}{ }^{\prime} \ldots x_{n}{ }^{\prime}\right)=K_{2 n}\left(x_{1} \ldots x_{n} \mid x_{1}{ }^{\prime} \ldots x_{n}{ }^{\prime}\right)+\sum_{\substack{i_{1} \ldots i_{n} \\ j_{1} \ldots j_{n}}} F\left(x_{i_{1}} x_{j_{1}}{ }^{\prime}\right) \ldots F\left(x_{i_{n}} x_{j_{n}}{ }^{\prime}\right)
$$


results. The last term in (4.11) can be identified with $\varphi_{2 n}^{0 f}$ as it is just the vacuum expectation value of the generalized free field (2.20) with bare groundstate. Then Eq. (1.23) can be written in components in the diagonal approximation (4.2)

$$
\begin{aligned}
\varphi_{n}\left(x_{1} \ldots x_{n} \mid a\right)=\int \varphi_{2 n}^{0 f}\left(x_{1} \ldots x_{n} \mid x_{1}{ }^{\prime} \ldots x_{n}{ }^{\prime}\right) & \sigma_{n}{ }^{r}\left(x_{1}{ }^{\prime} \ldots x_{n}{ }^{\prime} \mid a\right) \mathrm{d}^{4} x_{1}{ }^{\prime} \ldots \mathrm{d}^{4} x_{n}{ }^{\prime} \\
& +\int K_{2 n}\left(x_{1} \ldots x_{n} \mid x_{1}{ }^{\prime} \ldots x_{n}{ }^{\prime}\right) \sigma_{n}{ }^{r}\left(x_{1}{ }^{\prime} \ldots x_{n}{ }^{\prime} \mid a\right) \mathrm{d}^{4} x_{1}{ }^{\prime} \ldots \mathrm{d}^{4} x_{n}{ }^{\prime} .
\end{aligned}
$$

Equation (4.12) can be solved by applying the $\mathfrak{M}$-transform. For this we define symbolically

$$
\mathfrak{M}_{n} \varphi_{n}\left(x_{1} \ldots x_{n} \mid a\right):=m_{n}{ }^{r}\left(p_{1} \ldots p_{n-1} \mid I_{a}\right), \quad \mathfrak{M}_{n} \sigma_{n}{ }^{r}\left(x_{1}{ }^{\prime} \ldots x_{n}{ }^{\prime} \mid a\right):=s_{n}{ }^{r}\left(q_{1} \ldots q_{n-1} \mid I_{a}\right)
$$

and

$$
\mathfrak{M}_{n} K_{2 n}\left(x_{1} \ldots x_{n} \mid x_{1}^{\prime} \ldots x_{n}^{\prime}\right) \mathfrak{M}_{n}^{-1}=\Re_{2 n}\left(p_{1} \ldots p_{n-1} \mid q_{1} \ldots q_{n-1}\right) .
$$

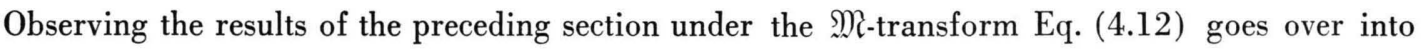

$$
\begin{aligned}
m_{n}{ }^{r}\left(p_{1} \ldots p_{n-1} \mid I_{a}\right)=\varrho\left(p_{1}\right) & \ldots \varrho\left(I_{a}-p_{n-1}\right) s_{n}{ }^{r}\left(p_{1} \ldots p_{n-1} \mid I_{a}\right) \\
& +\int \Re_{2 n}\left(p_{1} \ldots p_{n-1} \mid q_{1} \ldots q_{n-1}\right) s_{n}{ }^{r}\left(q_{1} \ldots q_{n-1} \mid I_{a}\right) \mathrm{d}^{4} q_{1} \ldots \mathrm{d}^{4} q_{n-1}
\end{aligned}
$$

or

$$
\begin{aligned}
s_{n}{ }^{r}\left(p_{1} \ldots p_{n-1} \mid I_{a}\right)=\varrho^{-1}\left(p_{1}\right) & \ldots \varrho^{-1}\left(I_{a}-p_{n-1}\right) m_{n}^{r}\left(p_{1} \ldots p_{n-1} \mid I_{a}\right) \\
& +\int \hat{K}_{2 n}\left(p_{1} \ldots p_{n-1} \mid q_{1} \ldots q_{n-1}\right) s_{n}^{r}\left(q_{1} \ldots q_{n-1} \mid I_{a}\right) \mathrm{d}^{4} q_{1} \ldots \mathrm{d}^{4} q_{n-1}
\end{aligned}
$$

with

$$
\hat{K}_{2 n}\left(p_{1} \ldots p_{n-1} \mid q_{1} \ldots q_{n-1}\right):=\varrho^{-1}\left(p_{1}\right) \ldots \varrho^{-1}\left(I_{a}-p_{n-1}\right) \AA_{2 n}\left(p_{1} \ldots p_{n-1} \mid q_{1} \ldots q_{n-1}\right) .
$$

Therefore, by this operation the first order integral Eq. (4.12) is changed into the second order integral Eq. (4.17). Integral equations of the second order can be solved by standard methods at least formally. So the Neumann series solution of (4.17) gives

$$
\begin{aligned}
& s_{n}^{r}\left(p_{1} \ldots p_{n-1} \mid I_{a}\right) \\
& \quad=\sum_{l=0}^{\infty} \int \hat{K}_{2 n}\left(p_{\mathbf{1}} \ldots p_{n-1} \mid q_{1} \ldots q_{n-1}\right)^{l} \varrho^{-1}\left(q_{1}\right) \ldots \varrho^{-1}\left(I_{a}-q_{l-1}\right) m_{n}{ }^{r}\left(q_{1} \ldots q_{n-1} \mid I_{a}\right) \mathrm{d}^{4} q_{1} \ldots \mathrm{d}^{4} q_{n-1}
\end{aligned}
$$

and from this results the scalar product

$$
\begin{array}{r}
\langle\mathfrak{I}(j, b) \mid \Im(j, a)\rangle=\langle b \mid a\rangle=\delta\left(I_{b}-I_{a}\right) \sum_{n l} \int m_{n}^{r}\left(p_{1} \ldots p_{n-1} \mid I_{b}\right) \times \hat{K}_{2 n}\left(p_{1} \ldots p_{n-1} \mid q_{1} \ldots q_{n-1}\right) \\
\cdot \varrho^{-1}\left(q_{1}\right) \ldots \varrho^{-1}\left(I_{a}-q_{n-1}\right) m_{n}^{r}\left(q_{1} \ldots q_{n-1} \mid I_{a}\right) \mathrm{d}^{4} q_{1} \ldots \mathrm{d}^{4} q_{n-1} \mathrm{~d}^{4} p_{1} \ldots \mathrm{d}^{4} p_{n-1} .
\end{array}
$$

For practical applications it is convenient to postulate an appropriate two point function $F\left(x, x^{\prime}\right)$ and to calculate the higher point function subsequently by (4.9). Then the kernel (4.10) is well defined and can be obtained by pure integrations. From this follows that also $\hat{K}_{2 n}$ is well defined and that (4.20) can finally be evaluated directly.

As (4.20) shall be applied mainly to scattering problems the normalization and the scalar product formation for ingoing or outgoing free cluster states is of interest. We give a short discussion of this problem by using the explicit state representation (1.6). Especially we consider a bound state defining a relativistic cluster. For this relativistic cluster we in troduce the cluster creation operator

$$
A^{+}(a):=\sum_{n=0}^{\infty} \int \sigma_{n}\left(a_{1} \ldots x_{n} \mid a\right) \bar{T} \Psi_{a_{1}}\left(x_{1}\right) \ldots \Psi_{a_{n}}\left(x_{n}\right) \mathrm{d}^{4} x_{1} \ldots \mathrm{d}^{4} x_{n}
$$

and write

$$
|a\rangle=A^{+}(a)|0\rangle \text {. }
$$

Due to (1.26) this creation operator can be expressed also by

$$
A^{+}(a):=\sum_{n=0}^{\infty} \int \sigma_{n}^{r}\left(x_{1}^{\alpha_{1}} \ldots x_{n}^{a_{n}} \mid a\right): \Psi_{a_{1}}\left(x_{1}\right) \ldots \Psi_{a_{n}}\left(x_{n}\right): \mathrm{d}^{4} x_{1} \ldots \mathrm{d}^{4} x_{n}
$$


and the connection between $\left\{{\sigma_{n}}^{r}\right\}$ and $\left\{\varphi_{n}{ }^{r}\right\}$ is given by the functional equation (1.23) with (1.24). Denoting the cluster quantum numbers by $\left\{\Re_{i}\right\}$ in ordinary Hilbert space a many cluster state is given by

$$
\left|\Re_{1} \ldots \Re_{n}\right\rangle:=A^{+}\left(\Re_{1}\right) \ldots A^{+}\left(\Re_{n}\right)|0\rangle
$$

while in functional space we write by definition according to ${ }^{16}$

$$
\left|\Phi\left(j, \Re_{1} \ldots \Re_{n}\right)\right\rangle:=\mathfrak{U}^{+}\left(\Re_{1}\right) \ldots \mathfrak{U}^{+}\left(\Re_{n}\right)\left|\varphi_{0}\right\rangle
$$

for the same cluster configuration. Then it can be verified easily, that in the diagonal approximation with $\Psi(x) \equiv x$ for brevity the relation

$$
\begin{aligned}
\varphi_{n_{1}}\left(x_{1}{ }^{1} \ldots x_{n_{1}}^{1} \mid \Re_{1}\right) \ldots & \varphi_{n_{k}}\left(x_{1}{ }^{k} \ldots x_{n_{k}}^{k} \mid \AA_{k}\right) \\
= & \int\left\langle 0\left|: x_{1}{ }^{1} \ldots x_{n_{1}}^{1}: \ldots: x_{1}{ }^{k} \ldots x_{n_{k}}^{k}:: y_{1}{ }^{1} \ldots y_{n_{1}}^{1}: \ldots: y_{1}{ }^{k} \ldots y_{n_{k}}^{k}:\right| 0\right\rangle \\
& \times \sigma_{n_{1}}^{r}\left(y_{1}{ }^{1} \ldots y_{n_{1}} \mid \Re_{1}\right) \ldots \sigma_{n_{k}}^{r}\left(y_{1}{ }^{k} \ldots y_{n_{k}}^{k} \mid \Re_{k}\right) \mathrm{d}^{4} y_{1}{ }^{1} \ldots \mathrm{d}^{4} y_{n_{k}}^{k}
\end{aligned}
$$

holds. We assume now that the different cluster are located at different regions in space as it is required for the initial or final configurations of scattering experiments. This means $\left|\mathfrak{x}_{a}{ }^{i}-\mathfrak{x}_{\beta}{ }^{j}\right| \gg 1$ and $\left|\mathfrak{y}_{a}{ }^{i}-\mathfrak{y}_{\beta}{ }^{j}\right|$ $\gg 1$ with $1 \leqq i, j \leqq k, 1 \leqq \alpha \leqq n_{i}, 1 \leqq \beta \leqq n_{j}$. Under this assumption the cluster decomposition property can be applied ${ }^{17}$ leading to the separated systems

$$
\varphi_{n_{i}}\left(x_{1}^{i} \ldots x_{n_{i}}^{i} \mid \Re_{i}\right)=\int \varphi_{2 n_{i}}^{0}\left(x_{1}^{i} \ldots x_{n_{i}}^{i} \mid y_{1}^{i} \ldots y_{n_{i}}^{i}\right) \sigma_{n_{i}}^{r}\left(y_{1}^{i} \ldots y_{n_{i}}^{i} \mid \Re_{i}\right) \mathrm{d}^{4} y_{1}{ }^{i} \ldots \mathrm{d}^{4} y_{n_{i}}^{i}
$$

$1 \leqq i \leqq k$. But these systems are just the normalization conditions for the single cluster states. Thus a general many cluster state of asymptotic distant clusters is normalized if its single components are normalized, and the normalization condition for many cluster states does not lead to new complication. Due to their localization the cluster states are wave packets. Therefore, they are not in a momentum representation and the scalar product of cluster states is more sophisticated. But this we will not discuss here in details as in principle this is treated in the theory of asymptotic states by Haag ${ }^{18}$.

Finally a remark about the selfconsistency problem has to be made. It has been already stressed that, if the normal transformation is defined only by (1.20), it cannot be applied to expressions like (1.5) or to the further reduction of (4.1). On the other hand the explicit calculation of states is inavoidable, and an indispensible condition is just the further reduction of (4.1). This can be performed only if normalordering can be defined independently of (1.20). The only way of doing this is to make additional assumptions about the spinor field operator. But these assumptions have to be in accordance with the general dynamical equations of the theory, namely the functional equations for the functionals $|\mathfrak{I}(j, b)\rangle$. As it is assumed that the vacuum expectation values suffice for securing the existence of the underlying field theory one has to conclude that the dynamical equations for the functionals $|\mathfrak{T}(j, b)\rangle$ are satisfied selfconsistently if this is true for the $|\mathfrak{I}(j, 0)\rangle$ functional. Therefore, in proving selfconsistency one can restrict oneself to discuss $|\mathfrak{I}(j, 0)\rangle$ and its equations. Assuming an expansion like (3.4) for the field operator, the only quantity which can be varied freely is the scalar spectral function $\varrho(m)$. On the other hand the complete functional Eq. (4.4) has to be satisfied. It is obvious that in general the function $\varrho(m)$ will not suffice to achieve this. Therefore, a more flexible ansatz has to be made. This ansatz has to secure the possibility of normalordering as well as the selfconsistency of (4.4). Assuming instead of (3.4) the general functional connection

$$
\left.\Psi_{a}(x)=\sum_{n=1}^{\infty} \int C \underset{a}{\left(x y_{1} \ldots y_{n}\right.} m_{1}^{\beta_{n}} \ldots m_{n}^{2}\right) \Psi_{\beta_{1}}\left(y_{1} m_{1}{ }^{2}\right) \ldots \Psi_{\beta_{n}}\left(y_{n} m_{n}{ }^{2}\right) \mathrm{d}^{4} y_{1} \ldots \mathrm{d}^{4} y_{n} \mathrm{~d} m_{1}{ }^{2} \ldots \mathrm{d} m_{n}{ }^{2}
$$

one can hope to satisfy simultaneously both conditions. But obviously by using a nonlinear functional for the connection between the generalized free field operators and the spinor field the calculations will turn out to be very trouble-some and the proof of selfconsistency seems although possible to be very hard. Therefore, functional quantum seems to be the appropriate approach to the problem as in this version the selfconsistency proof is not required at the beginning. 


\section{Acknowledgements}

I am indebted to Dipl.-Phys. D. Englert, Dipl.-Phys. G. Haegele and Dr. K. Scheerer for controlling my calculations and discussing the results.

1 W. Heisenberg, An Introduction to the Unified Theory of Elementary Particles, Wiley and Sons, London 1967.

2 H. P. Dürr, Quanten und Felder, Vieweg-Verlag, Braunschweig 1971, p. 125, ed. H. P. Dürr.

3 W. Heisenberg, Z. Phys. 120, 513, 678 [1942-43].

4 H. Stumpf, Ann. Phys. Leipzig 11, 138 [1963].

5 H. Stumpf, Ann. Phys. Leipzig 13, 294 [1964].

6 H. Stumpf, Acta Phys. Austr. Suppl. 9, 195 [1972].

7 H. Stumpf, Z. Naturforsch. 27 a, 1058 [1972].

8 H. Stumpf, Quanten und Felder, Vieweg-Verlag, Braunschweig 1971, p. 189, ed. H. P. Dürr.

9 H. Stumpf, Z. Naturforsch. 26 a, 1730 [1971].

10 K. Nishijima, Progr. Theor. Phys. 10, 549 [1953].
$11 \mathrm{H}$. Stumpf and K. Scheerer, Z. Naturforsch. in preparation.

12 D. Englert and H. Stumpf, Z. Naturforsch. 29 a, 376 [1974].

13 D. Englert, Rep. Math. Phys. 5, 337 [1974].

14 H. Stumpf, D. Englert, and W. Philipp, Z. Naturforsch. in preparation.

15 K. Dammeier, Z. Naturforsch. 27 a, 1042 [1972].

16 H. Stumpf, Z. Naturforsch. 29 a, 549 [1974].

17 P. F. Streater and A. S. Wightman, Die Prinzipien der Quantenfeldtheorie, Hochschultaschenbücher 435/435 a, Bibliograph. Inst. Mannheim/Zürich 1969.

18 R. Yost, General Theory of Quantized Fields, American Math. Soc. Publications 1963. 